\title{
GCU
}

Glasgow Caledonian

University

University for the Common Good

\section{Low complexity classification of power asset faults for real time loT-based diagnostics}

\author{
Salimy, Alireza; Mitiche, Imene; Boreham, Philip ; Nesbitt, Alan; Morison, Gordon
}

Published in:

2020 IEEE Global Conference on Artificial Intelligence and Internet of Things, GCAloT 2020

DOI:

10.1109/GCAIoT51063.2020.9345846

Publication date:

2021

Document Version

Author accepted manuscript

Link to publication in ResearchOnline

Citation for published version (Harvard):

Salimy, A, Mitiche, I, Boreham, P, Nesbitt, A \& Morison, G 2021, Low complexity classification of power asset faults for real time loT-based diagnostics. in 2020 IEEE Global Conference on Artificial Intelligence and Internet of Things, GCAloT 2020., 9345846, 2020 IEEE Global Conference on Artificial Intelligence and Internet of Things, GCAloT 2020, IEEE, 2020 IEEE Global Conference on Artificial Intelligence and Internet of Things, 12/12/20. https://doi.org/10.1109/GCAloT51063.2020.9345846

\section{General rights}

Copyright and moral rights for the publications made accessible in the public portal are retained by the authors and/or other copyright owners and it is a condition of accessing publications that users recognise and abide by the legal requirements associated with these rights.

Take down policy

If you believe that this document breaches copyright please view our takedown policy at https://edshare.gcu.ac.uk/id/eprint/5179 for details of how to contact us. 


\section{GCU}

Glasgow Caledonian

University

University for the Common Good

\section{Low Complexity Classification of Power Asset Faults for Real Time loT-based Diagnostics \\ Salimy, Alireza; Mitiche, Imene; Boreham, Philip ; Nesbitt, Alan; Morison, Gordon}

Publication date:

2020

Document Version

Publisher's PDF, also known as Version of record

Link to publication in ResearchOnline

Citation for published version (Harvard):

Salimy, A, Mitiche, I, Boreham, P, Nesbitt, A \& Morison, G 2020, 'Low Complexity Classification of Power Asset Faults for Real Time loT-based Diagnostics'.

\section{General rights}

Copyright and moral rights for the publications made accessible in the public portal are retained by the authors and/or other copyright owners and it is a condition of accessing publications that users recognise and abide by the legal requirements associated with these rights. 


\title{
Low Complexity Classification of Power Asset Faults for Real Time IoT-based Diagnostics
}

\author{
Alireza Salimy*, Imene Mitiche*, Philip Boreham ${ }^{\dagger}$ \\ Alan Nesbitt* and Gordon Morison* \\ * School of Computing, Engineering and Built Environment \\ Glasgow Caledonian University \\ Glasgow, United Kingdom \\ $\dagger$ Innovation Centre for Online Systems \\ Doble Engineering \\ Bere Regis, United Kingdom
}

\begin{abstract}
This paper investigates a new application of Capsule Neural Network (CapsNet), in combination with Constant-Q Transform (CQT), for insulation fault signal detection in High Voltage (HV) power plants. First, a mapping from insulation fault time-series signals to time-frequency images is obtained using the CQT, providing both time and frequency information. Different ways of exploiting the resulting complex-valued CQT are proposed; the CQT magnitude as a 1-channel image and the real-imaginary values of the CQT as a 2-channel image. This paper presents novel work in HV condition monitoring by utilising the CQT and CapsNet methods. Feature extraction and classification, from the produced CQT spectrum, is performed by CapsNet and the Residual Neural Network (ResNet). A performance comparison between both models, shows that CapsNet outperforms the ResNet in terms of classification accuracy with lower computation. The reduced computation and improved classification accuracy proves ideal, for system implementation on an edge embedded device incorporated in an Internet of Things (IoT) arrangement.
\end{abstract}

\section{INTRODUCTION}

Condition monitoring is crucial to power generation, as the High-Voltage (HV) and mechanical equipment used to carry out power generation are prone to faults. When these faults are incurred the fallout causes many unwanted issues, such as; costly power outages of electrical network systems, safety hazards, fines, and legal issues [1]. Condition monitoring not only provides the ability to avoid these problems through fault prevention, it also creates a more efficient maintenance process by preventing over maintenance, this maximises business profits when implemented. Electromagnetic Interference (EMI) is a measurement used to detect Partial Discharge (PD) in HV systems [2]. PD measurement and analysis methods are commonly used for monitoring insulation deterioration in electrical equipment [3], if such deteriorations are left uncorrected they may cause system failures. The EMI fault diagnostic process involves the measurement of the EMI signals which undergo manual expert analysis to identify the presence of faults in HV equipment [4], this method is carried out online and does not require the HV system to be powered off. The ability to use an Internet of Things (IoT) based, automated, accurate, and continuous condition monitoring systems would provide the highly beneficial outcomes of online precise and early fault detection. Therefore, this research aims to create an intelligent system, by utilising Artificial Intelligence (AI) techniques, to be deployed on an edge embedded device within an IoT system. The system would reduce dependence on staff and have continuous online functionality while simultaneously providing both $\mathrm{HV}$ condition monitoring experts and nonexperts the ability to confidently interpret the operational data. The creation of this system consists of two sections; data preprocessing and classification. The former consists of finding the Constant-Q Transform (CQT) of the EMI signals using methods found in [5], the produced CQT spectogram consists of high-frequency resolution electrical discharge signals at individual time steps. The latter consists of implementing a Capsule Neural Network (CapsNet) architecture to classify the collected EMI signals, there are 5 fault classes which are prevalent in the EMI data. CapsNet was originally introduced by [6], with their first implementations being used for the handwritten digit detection and image reconstruction problem. They showed not only the ability to perform better than the state-of-the-art methods but also included the ability to recognise and classify overlapping digits. Since their introduction, CapsNet architectures have been used in several classification problems [7] [8] which demonstrate that CapsNet outperforms the state-of-the-art Deep Learning networks. The conclusions drawn from the research exploring these architectures provide a strong basis to explore further benefits of CapsNet in various applications.

Previous work in $\mathrm{HV}$ condition monitoring with Deep Learning systems has been carried out in [1] [9], which employed Convolutional Neural Network (CNN) for fault classification. Work carried out in [10] outlined the benefits of approaching the HV condition monitoring problem with an IoT based solution, using such an approach allowed for interconnected sensors and monitoring equipment with realtime analysis, allowing quick fault location detection and continuous monitoring of several sites. [11] Shows the dynamic nature of an IoT approach showing that this approach in the field can be designed to be extendable and versatile to be updated with regards to the requirements. IoT based works in the $\mathrm{HV}$ asset monitoring field have added more to the 
automation of the condition monitoring task, allowing the previously discussed benefits of having a continuous monitoring approach to be more feasible, showing that by combining AI and IoT technologies, systems can be made to utilise the most efficient approach benefiting the health and safety of workers while also providing benefits for business. The works mentioned have provided good arguments to explore the use of $\mathrm{AI}$ in an IoT approach to HV condition monitoring. The approach in this study provides a comparison between the Residual Neural Network (ResNet) used in [9] and the CapsNet approach, on the CQT images. With the system which provides the most desirable results, the system with the highest accuracy and lowest computation, being implemented on an IoT embedded device. CapsNet attempts to improve upon CNNs by replacing the max-pooling layer, which loses spatial information, using dynamic routing and replacing the scalar outputs of CNNs with vector output Capsules [6]. This study explores the benefits produced by this alternative approach in $\mathrm{HV}$ condition monitoring and finds that the novel approach outperforms previously implemented methods in the field. Fig. 1 shows how the IoT system can be implemented, this study will focus on the operations outlined inside the edge embedded device.

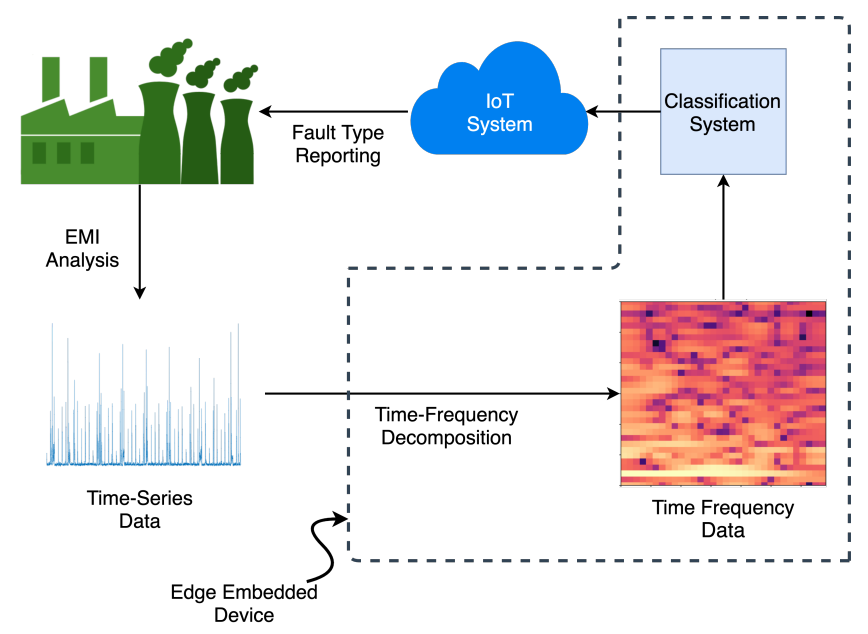

Fig. 1: Overall System, Showing Data Collection from HV Plants Edge Embedded Device Operations and IoT communication

The structure of the remainder of this paper is outlined as follows: Section II- Introduces the CQT and explains its derivation, Section III- Introduces and explains CapsNet and their functions, Section IV- Provides a description of the data and experimental methods, including the obtained results, Section V-Discusses and concludes the proposed methods, providing some insight to future work.

\section{COnStant-Q TRAnsform (CQT)}

First, the notation is defined for scalars as lowercase and uppercase, vectors as bold lowercase, and matrices as bold uppercase. The CQT consists of a bank of filters that have centre frequencies $f_{k}$ defined as:

$$
f_{k}=f_{\min } \times 2^{k / P}
$$

where $P$ is the number of filters per octave and $k$ is the spectral component of CQT. The frequency $f_{\min }$ must be chosen to satisfy the Nyquist criteria [5]. The window length $M_{k}$ varies inversely with frequency and is calculated as:

$$
M_{k}=\frac{f_{s}}{\Delta f_{k}}
$$

$f_{s}$ is the sampling frequency and $\Delta f_{k}=f_{k}\left(2^{1 / P}-1\right)$ is the bandwidth. The ratio of the frequency to the bandwidth is required to be a constant called the $Q$ factor:

$$
Q=\frac{f_{k}}{\Delta f_{k}}
$$

Based on these constraints, the CQT spectrum $\mathbf{X}$ for the $k^{t h}$ component of a signal $\mathrm{x}$ is calculated as:

$$
\mathbf{X}_{k}=\frac{1}{M_{k}} \sum_{n=0}^{M_{k}-1} \boldsymbol{\Omega}_{(k, n)} \mathbf{x}_{n} \cdot e^{\frac{-j 2 \pi Q n}{M_{k}}}
$$

$\boldsymbol{\Omega}_{(k, n)}$ represents the Hamming window [12] at the $k^{t h}$ and $n^{t h}$ spectral and temporal components respectively.

The constant $\mathrm{Q}$ factor helps to achieve a time-frequency spectogram with high-frequency resolution at low frequencies and high temporal resolution at high frequencies. This property is the main reason for CQT application to audio signals as it better reflects the resolution in the human auditory system [13]. EMI experts often analyse the measured signals by listening to the audio version of the signals [14]. The CQT of the electrical discharge signals at each time step produces a complex 2D input for the CapsNet, this is used to provide a 2channel complex input and a 1-channel magnitude input, used in the implementations outlined in IV. Fig. 2 illustrates the example CQT output of two different insulation fault signals used in this work, where the first CQT image shows the magnitude, followed by the real then the imaginary parts of the CQT.

\section{CAPSUlE NETWORKS}

CapsNet algorithms are designed to be an alternative to traditional CNNs, they have shown to work well with classification problems [15]. This performance enhancement is achieved by providing the ability to communicate through layers while holding information about spatial relationships between features. Most CNNs attempt to reduce the spatial size of the representations by pooling to decrease the computation of the network however, this loses some valuable spatial information. The loss in spatial information comes from pooling after the convolutional layer. CapsNet attempts to overcome this loss by implementing dynamic routing, the design is based on human vision and its capability to ignore irrelevant details [6] [16]. CapsNets differ from standard CNNs due to their structure, in contrast to the latter their layers are divided into 

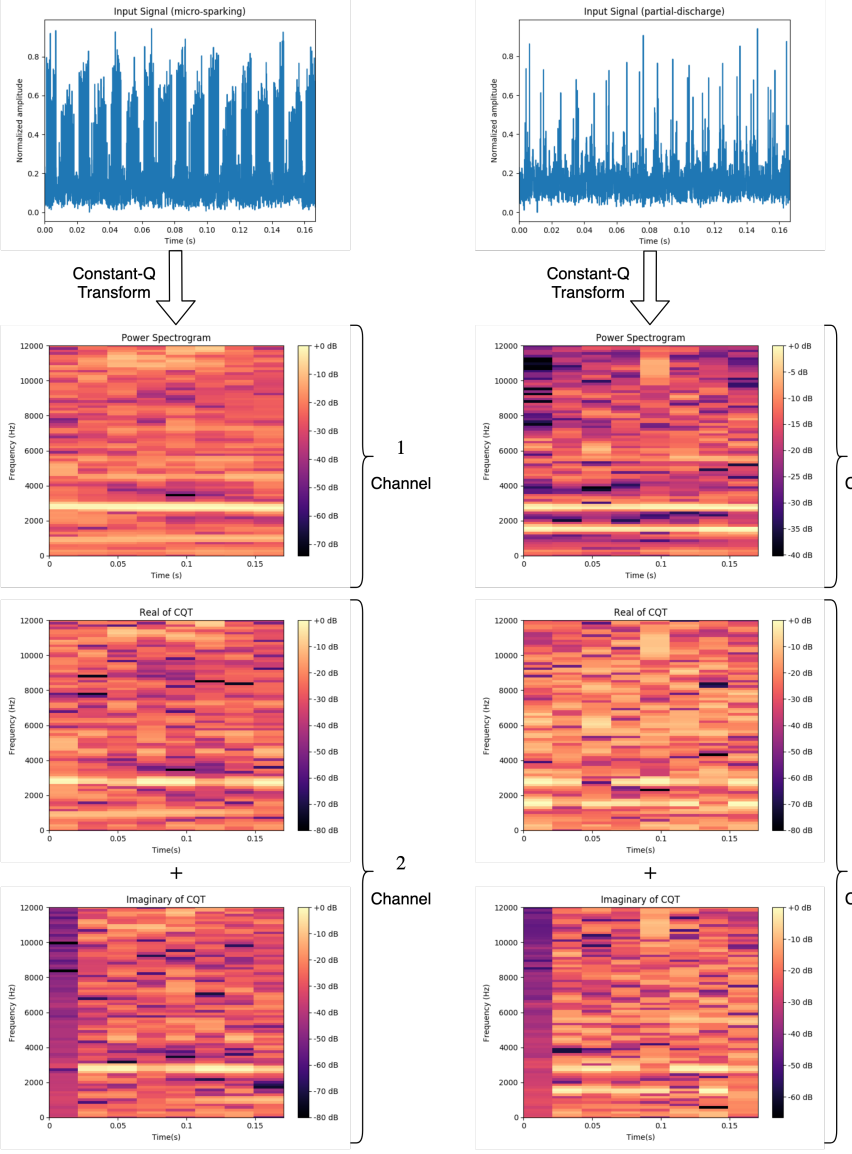

Fig. 2: CQT Output (Spectrogram, Real and Imaginary Respectively) of Micro-sparking (left) and PD (right) Signals

many groups of neurons which are called 'Capsules'. The Capsules carry out voting by outputting a vector that defines the probability of a feature being present and its orientation, votes are accounted for and are used to decide on feature presence. The routing process in CapsNet provides many benefits, as it allows communication and agreement between Capsules, and is crucial to its performance. Tying Section II with this section, the overall implementation is illustrated in Fig. 3, which will analyse multiple EMI signal types that will be described in Section IV. The building components of CapsNet are described in detail as follows.

\section{A. Convolution}

Convolutional layers are used to extract a feature map from an input image. Equation (5) shows the properties of a convolutional layer, $\mathbf{X}$ representing the input CQT image, $\mathbf{K}$ being the filter of size $x_{1} \times x_{2}$ and letting $L$ and $N$ to be the length and width of the image respectively, these values were fixed at $L=84$ and $N=8$ as they provided the best computation and accuracy trade-off. Where $r=0,1, \ldots . ., N-1, z=0,1, \ldots . ., L-1$. After the addition of the bias $q$, the layer outputs are created to be non-linear, this is achieved by using an activation function $f(\cdot)$. The input information may also consist of more than a single channel
$(C \geq 1), C$ represents the number of channels in the input data.

$$
\begin{aligned}
(\mathbf{X} * \mathbf{K})_{m, n}=f\left(\sum_{m=0}^{x_{1}-1} \sum_{n=0}^{x_{2}-1} \sum_{h=1}^{C}\right. \\
\left.\mathbf{X}_{(m+r, n+z, h)} \mathbf{K}_{(m, n, h)}+q\right)
\end{aligned}
$$

The CapsNet architecture described in this paper was proposed in [6]. It consists of two convolutional layers; 1) Conv2D_1 layer is used to convert the original channel pixel intensities to activity values for local feature detectors, the result is then the input of 'Primary Caps' which is the first Capsule layer. With activation function ReLU: $f(\cdot)=\max (0, x), 2)$ Conv2D_2 layer found in all Capsule layers, with activation function Linear: $f(\cdot)=x$.

\section{B. Capsule input and output vectors}

The total input of the Capsule $\mathbf{s}_{b}$, input $\mathbf{s}$ of Capsule $b$ in the next layer ${ }^{1}$, is calculated using (7) by finding the weighted sum of all prediction vectors $\hat{\mathbf{u}}_{b \mid a}$, which are computed using the 2 Capsule outputs of the previous layer $\mathbf{u}_{a}$ and a transformation channel matrix $\mathbf{W}_{a b}$, as shown in (6).

$$
\begin{gathered}
\hat{\mathbf{u}}_{b \mid a}=\mathbf{W}_{a b} \mathbf{u}_{a} \\
\mathbf{s}_{b}=\sum_{a} c_{a b} \hat{\mathbf{u}}_{b \mid a}
\end{gathered}
$$

All of the produced prediction vectors are multiplied by a corresponding coupling co-efficient $c_{a b}$, the sum of the resultant vectors gives the total input to the next Capsule layer. The coupling co-efficients are produced by 'dynamic routing' [6]. The coupling co-efficients between layer $a$ and $b$ sum to 1 and are found through a 'routing softmax' with initial logits $g_{a b}$, equivalent to the log prior probabilities that Capsule $a$ should be coupled to Capsule $b$, this relationship is shown in (8). This can be learned concurrently with the learning of the various weights, the log priors $\left(g_{a b}\right)$ are solely dependant on the type and location of both Capsules in layer $a$ and $b$, with $d$ being the class Capsule of the feature.

$$
c_{a b}=\frac{\exp \left(g_{a b}\right)}{\sum_{d} \exp \left(g_{a d}\right)}
$$

The original coupling co-efficients are redefined per iteration, this is carried out by measuring the agreement between the current output $\mathbf{v}_{b}$ and the prediction vectors $\hat{\mathbf{u}}_{b \mid a}$. This agreement is the corresponding scalar product of $\mathbf{v}_{b}$ and $\hat{\mathbf{u}}_{b \mid a}$, this value is treated as a log likelihood and is subsequently added to the initial logit $g_{a b}$, outlined in (9). Producing new values for the coupling co-efficients connecting a Capsule in layer $a$ to layer $b$.

$$
g_{a b} \longleftarrow g_{a b}+\hat{\mathbf{u}}_{b \mid a} \cdot \mathbf{v}_{b}
$$

\footnotetext{
${ }^{1}$ When discussing Capsule layers, $a$ and $b$ will be used to represent a Capsule in the present Capsule layer and a Capsule in the ensuing Capsule layer respectively.
} 


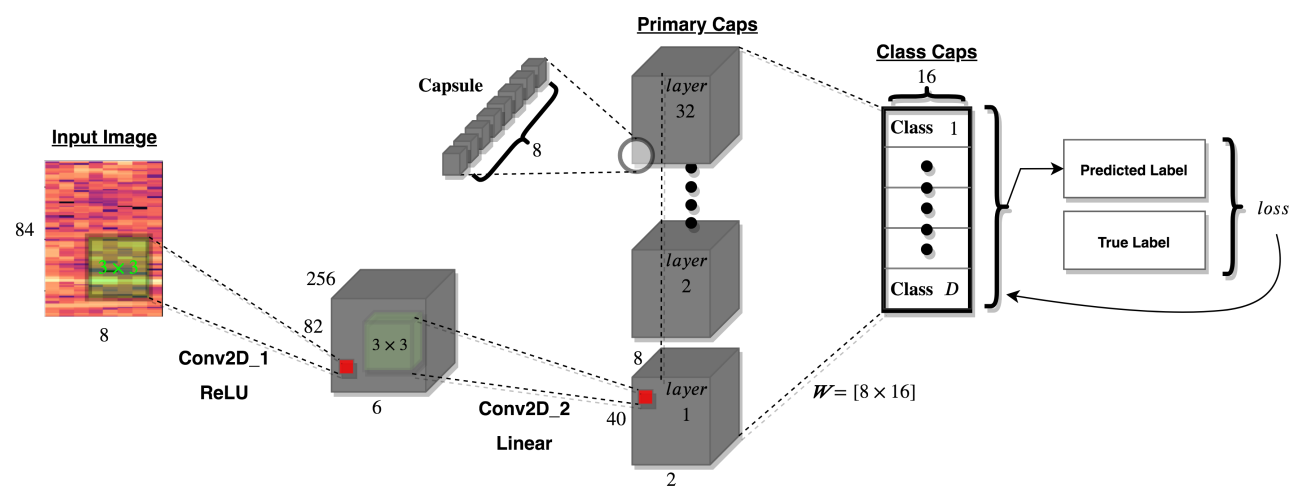

Fig. 3: CapsNet Architecture, Based on 1 Channel CQT Input

Capsules attempt to represent the probability of their features being present in input data by producing a vector, with magnitude $0 \rightarrow 1$. Vectors with a magnitude close to the value 1 correspond to Capsules which occur with high probability in the input and those with a value close to 0 correspond to Capsules which do not occur in the input. In order to meet this criterion a 'Squash' operation is calculated on $s_{b}$, to obtain the output vector $\mathbf{v}_{b}$ of Capsule $b$ as shown in (10). Fig. 4 shows the relationship between input and output vectors and the procedures to compute them.

$$
\mathbf{v}_{b}=\frac{\left\|\mathbf{s}_{b}\right\|^{2}}{1+\left\|\mathbf{s}_{b}\right\|^{2}} \frac{\mathbf{s}_{b}}{\left\|\mathbf{s}_{b}\right\|}
$$

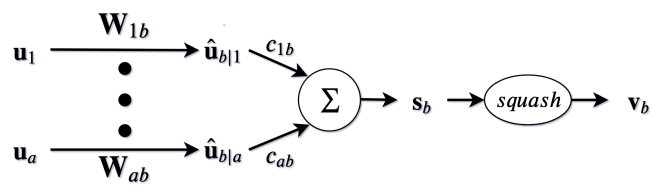

Fig. 4: Vector Input and Output Diagram, Showing Routing and Weighted Summation

\section{Dynamic routing}

Routing is one of the main contributors to the performance boost provided by CapsNet, it allows Capsule layers to communicate. Capsules in layers $a$ are given the ability to communicate with layers $b$, this is done by routing by agreement and in this paper the used algorithm is 'dynamic routing' [6] illustrated in procedure 1. The algorithm decides how to carry out this communication by changing the scalar weight $c_{a b}$ which multiplies the output vector from the lower Capsule layer, producing the input to the higher level layer. The weight $c_{a b}$ has several important properties; each weight applied is non-negative, the sum of all low-level Capsule weights is equal to 1 , the number of weights is equal to the number of higher level Capsules and the weights are updated at every iteration of dynamic routing.

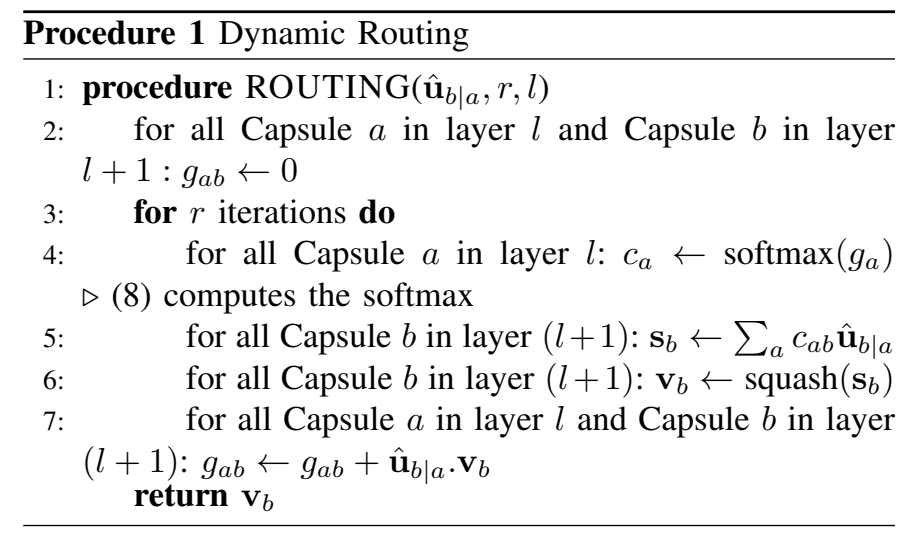

\section{CapsNet loss}

During training the total loss value is computed as:

$$
\begin{aligned}
\text { loss }=\sum_{d=1}^{D} \mathbf{t}_{d} \max & \left(0, p^{+}-\left\|\mathbf{v}_{d}\right\|\right)^{2} \\
& +\lambda\left(1-\mathbf{t}_{d}\right) \max \left(0,\left\|\mathbf{v}_{d}\right\|-p^{-}\right)^{2}
\end{aligned}
$$

where $D$ is the total number of classes, $\lambda$ is a constant used to produce numerical stability and is set to $\lambda=0.5$ along with $p^{+}=0.9$ and $p^{-}=0.1$ for the experiments in this paper, as recommended in [6]. The true label $\mathbf{t}_{d}$ is equal to 1 when an instance of class $d$ is present and it is equal to 0 when the class is not present. The $\lambda$ value is used as a down-weighting to avoid initial learning from shrinking the lengths of the activity vectors of all the class Capsules [6].

\section{EXPERIMENTAL SET-UP}

\section{A. The dataset}

The data used in this work were collected from realworld assets including generators, isolated phase bus, feed pumps, and transformers, operating at HV power plants. The data measurement was designed to gather information on potential insulation and mechanical faults occurring within assets to address the problem and allow trending for future decisions. The data are in form of time-resolved signals that were measured using the EMI technique [17], which follows 
TABLE I: Structure of the ResNet Model

\begin{tabular}{c|c|c|c|c} 
Layer & Kernel & Depth & Stride & Operation \\
\hline \hline 1 & $7 \times 7$ & 32 & 2 & Conv \\
\hline 2 & $3 \times 3$ & - & 1 & MaxPool \\
\hline 3 & $3 \times 3$ & 32 & 1 & Residual Block x 2 \\
\hline 4 & $3 \times 3$ & 64 & 1 & Residual Block x 2 \\
\hline 5 & $3 \times 3$ & 128 & 1 & Residual Block x 2 \\
\hline 6 & $3 \times 3$ & 256 & 1 & Residual Block x 2 \\
\hline 7 & \multicolumn{6}{|c}{ Average Pool, Fully Connected, SoftMax } \\
\hline
\end{tabular}

the Comite International Special des Perturbations Radioelectriques (CISPR) 16 standard [18], to measure the radiated or conducted EMI through high-frequency current transformer. The signals are sampled at a rate of 24000 samples per second and are analysed and labelled by an EMI expert to identify the type of occurring fault [19]. Labels are assigned, by EMI experts, to each signal providing four different insulation faults called arcing, corona, PD, and micro-sparking, and four non-fault measurement events such as process and random noise, data modulation, etc. The latter were grouped under one category as 'non-significant'. This provides a total of five categories to classify. The total number of arcing signals is 546, corona: 59, PD: 5057 , micro-sparking: 433 , and the remaining "non-significant" category contains 1873 signals.

\section{B. Fault classification using CapsNet}

The CQT of the collected EMI signals is used to obtain the time-frequency spectogram, which results in a $2 \mathrm{D}$ complex entity. Therefore, the authors propose two ways of exploiting the CQT spectogram. The first is to obtain the magnitude of the complex entity which results in a $L \times N$ image. However, this method discards phase-related information. The second approach is to exploit both the magnitude and phase information by separating the values of the real and imaginary parts providing a 2 channel image of dimension $L \times N \times 2$. The CapsNet model described in the previous section is used as a classifier for the EMI data, taking as its input the magnitude or the complex CQT. The performance of these two methods are compared along with a ResNet-18 version of implementation [20]. Here, four implementations are compared:

1) $|\mathbf{X}|$ input for CapsNet (1 channel).

2) $\mathbf{X}$ input for CapsNet (2 channel).

3) $|\mathbf{X}|$ input for ResNet-18 (1 channel).

4) $\mathbf{X}$ input for ResNet-18 (2 channel).

The employed CapsNet architecture is illustrated in Fig. 3 and a ResNet-18 architecture is provided in Table I. All models were implemented using Tensorflow [21] and Keras [22] as multi-class classifiers, where binary cross-entropy loss was used for ResNet and the margin loss described in 11 was used for the CapsNet. Adam optimiser was used for both networks over 100 epochs however, it was observed that the training loss did not improve after 50 epochs for CapsNet. The data presented in Section IV-A was split into $(70,15,15) \%$ for training, validation, and testing respectively. The training data was unbalanced, therefore automatic class weighting was set
TABLE II: Performance Comparison of CapsNet $(1 \& 2)$ and ResNet ( $3 \& 4)$. Best Performance is Presented in Bold Font.

\begin{tabular}{c|c|c|c} 
Implementation & Test accuracy \% & Training time & Total \# params \\
\hline 1 & $\mathbf{9 7 . 4}$ & $40 \mathrm{~min} 48 \mathrm{sec}$ & $2,231,040$ \\
2 & 96.8 & $40 \mathrm{~min} 50 \mathrm{sec}$ & $2,233,344$ \\
3 & 96.6 & $9 \mathrm{~h} 13 \min 15 \mathrm{sec}$ & $2,801,157$ \\
4 & 93.14 & $9 \mathrm{~h} 40 \mathrm{~min} 14 \mathrm{sec}$ & $2,801,445$
\end{tabular}

in Keras training. The proposed algorithms were trained and tested using an RTX 2080 Ti Graphics Processing Unit.

System performance is evaluated in terms of classification accuracy $(a c c)$ of the test data, defined as:

$$
\begin{gathered}
a c c=\frac{1}{H} \sum_{i=1}^{H} A\left(\hat{\mathbf{y}}_{i}=\mathbf{y}_{i}\right) \\
A\left(\hat{\mathbf{y}}_{i}=\mathbf{y}_{i}\right)= \begin{cases}1 & \hat{\mathbf{y}}_{i}=\mathbf{y}_{i} \\
0 & \text { otherwise }\end{cases}
\end{gathered}
$$

where $H$ is equal to the number of testing samples, $\hat{\mathbf{y}}$ is the prediction output vector and $\mathbf{y}$ is the ground truth label vector for all samples. In order to assess the computation performance of the networks, the training time and the total number of parameters per network are calculated. Each model training used the same computation resources (one GPU) under the same conditions.

\section{Results}

The classification accuracy, training time and the total number of parameters are presented in Table II for the implementations discussed in Section IV-B. It is observed that CapsNet outperforms the 18-layer ResNet in the classification task for a lower training time, although the number of total parameters is closely similar for both networks. The number of parameters is highest in the CapsNet dynamic routing step, as the Capsule inputs in the next Capsule layer are calculated through a transformation weight matrix on the output of the Capsule observed in the current Capsule layer (see (6) and algorithm 1). Since the log priors and weights during routing are updated in parallel, this brings a computation time benefit over ResNet. The routing is performed through 3 iterations and the CapsNet architecture is very shallow compared to the ResNet-18. The latter performs the forward pass and backpropagation in series which reduces training time, especially if the network architecture is deeper. The system with the greatest accuracy and lowest computation is chosen to be implemented on the embedded edge device in an IoT system, shown in Fig. 5, to monitor HV equipment conditions.

\section{CONClusion}

Insulation fault signal classification was performed using the CQT combined with CapsNet. The network achieved higher classification accuracy than the ResNet at lower computation time. It was demonstrated that the parallel weights and prior logs update during CapsNet training improve the training time. 


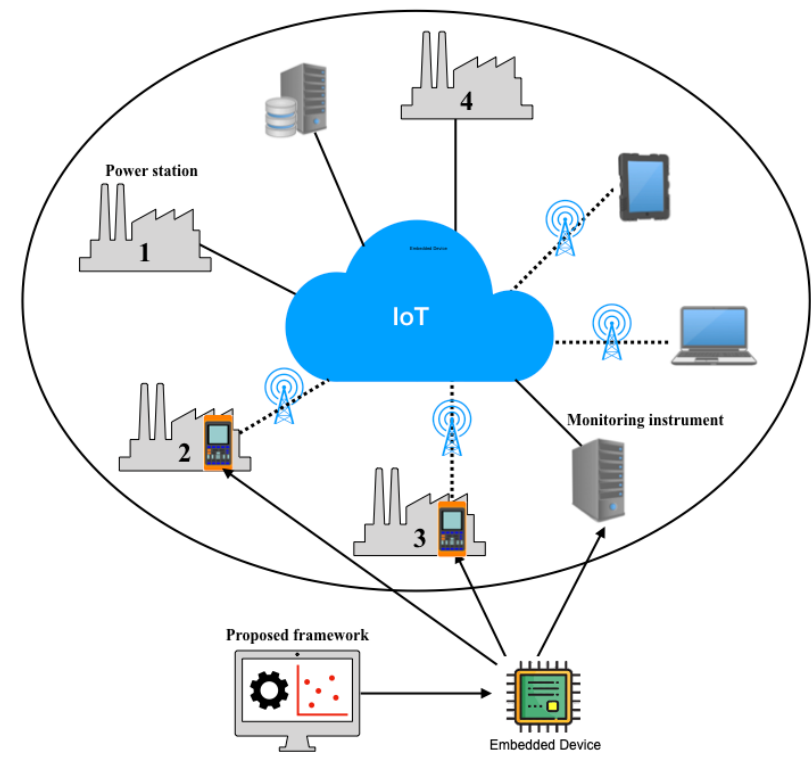

Fig. 5: IoT-Based HV Equipment Monitoring System

The high classification accuracy and lower computation of CapsNet proves it to be a feasible algorithm to be implemented in real-world applications, such as an edge embedded device in a IoT system used for machine health diagnostics in the power industry. Future research should investigate the inference time difference between both networks, in a hardware platform. Further studies could be conducted to implement the complex CQT with a complex-valued CapsNet [23], and explore the application of CapsNet to alternative forms of data for power system condition monitoring.

\section{REFERENCES}

[1] S. Barrios, D. Buldain, M. Comech, I. Gilbert, and I. Orue Sagarduy, "Partial discharge classification using deep learning methods-survey of recent progress," Energies, vol. 12, p. 2485, 2019.

[2] J. Timperley, D. Buchanan, and J. Vallejo, "Electric generation condition assessment with electromagnetic interference analysis," IEEE Transactions on Industry Applications, vol. PP, pp. 1-1, 2017.

[3] G. C. Montanari and A. Cavallini, "Partial discharge diagnostics: from apparatus monitoring to smart grid assessment," IEEE Electrical Insulation Magazine, vol. 29, pp. 8-17, 2013.

[4] J. E. Timperley and J. M. Vallejo, "Condition assessment of electrical apparatus with emi diagnostics," in 2015 IEEE Petroleum and Chemical Industry Committee Conference (PCIC), 2015, pp. 1-8.

[5] J. C. Brown, "Calculation of a constant q spectral transform," Acoustical Society of America, vol. 89, pp. 425-434, 1991.

[6] S. Sabour, N. Frosst, and G. E. Hinton, "Dynamic routing between capsules," in International Conference on Neural Information Processing Systems, 2017.

[7] P. Afshar, A. Mohammadi, and K. N. Plataniotis, "Brain tumor type classification via capsule networks," in 2018 25th IEEE International Conference on Image Processing (ICIP), 2018, pp. 3129-3133.

[8] M. E. Paoletti, J. M. Haut, R. Fernandez-Beltran, J. Plaza, A. Plaza, J. Li, and F. Pla, "Capsule networks for hyperspectral image classification," IEEE Transactions on Geoscience and Remote Sensing, vol. 57, pp. 2145-2160, 2019.

[9] I. Mitiche, M. D. Jenkins, P. Boreham, A. Nesbitt, and G. Morison, "Deep complex neural network learning for high-voltage insulation fault classification from complex bispectrum representation," in 2019 27th European Signal Processing Conference (EUSIPCO), 2019, pp. 1-5.

[10] J. H. Y. Cai, XQ. Huang, in Wireless Communication and Applications, 2012, pp. 44-57.
[11] T. Zhuang, M. Ren, X. Gao, M. Dong, W. Huang, and C. Zhang, "Insulation condition monitoring in distribution power grid via iot-based sensing network," IEEE Transactions on Power Delivery, vol. 34, pp. $1706-1714,2019$

[12] A. V. Oppenheim, R. W. Schafer, and J. A. Buck, Discrete-time signal processing. Upper Saddle River, N.J.: Prentice Hall, 1999.

[13] M. Todisco, H. Delgado, and N. Evans, "Constant q cepstral coefficients: A spoofing countermeasure for automatic speaker verification," Computer Speech Language, vol. 45, pp. 516-535, 2017.

[14] J. E. Timperley, "Detection of insulation deterioration through electrical spectrum analysis," in 6th Electrical/Electronical Insulation Conference (EIC), 1983, pp. 60-64.

[15] M. Yang, W. Zhao, J. Ye, Z. Lei, Z. Zhao, and S. Zhang, "Investigating capsule networks with dynamic routing for text classification," in Conference on Empirical Methods in Natural Language Processing, 2018.

[16] G. E. Hinton, S. Sabour, and N. Frosst, "Matrix capsules with EM routing," in International Conference on Learning Representations, 2018.

[17] J. E. Timperley and J. M. Vallejo, "Condition assessment of electrical apparatus with emi diagnostics," IEEE Transactions on Industrial Applications, vol. 53, pp. 693-699, 2017.

[18] "Cispr/cis/a-radio-interference measurements and statistical methods." International Electrotechnical Commission, Standard, Mar. 2015.

[19] J. E. Timperley, P. Longo, D. McKim, and J. M. Vallejo, "Electromagnetic interference data collection from bus coupler capacitors," in Electrical Insulation Conference, 2018, pp. 178-181.

[20] K. He, X. Zhang, S. Ren, and J. Sun, "Deep residual learning for image recognition," in IEEE Conference on Computer Vision and Pattern Recognition, 2016, pp. 770-778.

[21] M. Abadi, A. Agarwal, P. Barham, E. Brevdo, Z. Chen, C. Citro, G. S. Corrado, A. Davis, J. Dean, M. Devin, S. Ghemawat, I. Goodfellow, A. Harp, G. Irving, M. Isard, Y. Jia, R. Jozefowicz, L. Kaiser, M. Kudlur, J. Levenberg, D. Mané, R. Monga, S. Moore, D. Murray, C. Olah, M. Schuster, J. Shlens, B. Steiner, I. Sutskever, K. Talwar, P. Tucker, V. Vanhoucke, V. Vasudevan, F. Viégas, O. Vinyals, P. Warden, M. Wattenberg, M. Wicke, Y. Yu, and X. Zheng, "TensorFlow: Large-scale machine learning on heterogeneous systems," http://tensorflow.org/, 2015.

[22] F. Chollet et al., "Keras," https://github.com/fchollet/keras, 2015.

[23] X. Cheng, J. He, J. Hea, and H. Xu, "Cv-capsnet: Complex-valued capsule network," IEEE Access, vol. 7, pp. 85 492-85 499, 2019. 